\title{
Systematic review and meta-analysis: surgical reparative treatment for orthopedic patients with ankle fracture complicated by peripheral tissue injury
}

\author{
Xianxue Xia, Zhiqiang Yang, Yuan Zhang, Changgong Deng, Wei Zhang, Lu Chen \\ Department of Orthopedics, Affiliated Hospital of North Sichuan Medical College, Nanchong, China \\ Contributions: (I) Conception and design: X Xia, L Chen; (II) Administrative support: Z Yang; (III) Provision of study materials or patients: X Xia, \\ Z Yang, Y Zhang, C Deng, W Zhang, L Chen; (IV) Collection and assembly of data: All authors; (V) Data analysis and interpretation: Y Zhang, C \\ Deng, L Chen; (VI) Manuscript writing: All authors; (VII) Final approval of manuscript: All authors. \\ Correspondence to: Lu Chen. Department of Orthopedics, Affiliated Hospital of North Sichuan Medical College, 1 Zhaoyuan South Road, Shunqing \\ District, Nanchong 637000, China. Email: 18057819@qq.com.
}

\begin{abstract}
Background: This study aimed to apply meta-analysis to study the efficacy of surgical repair treatment on ankle fractures.

Methods: The Boolean logic search method was adopted to retrieve relevant randomized control trials (RCTs), with "Fracture", "Ankles", "Fractured Foot", "Fractures Merge", and "Surgical Treatment" as search terms. The databases PubMed, Medline, HowNet, and others were searched from the time of their establishment. The software Review Manager (Rev Man 5.3) was used for meta-analysis.

Results: A total of 10 references were included in the study, and most of them had low-risk bias (mediumhigh quality). The results of meta-analysis showed that after surgical repair treatment, gait was good, while the gait score of the non-surgical group was poor. The result was $\mathrm{Chi}^{2}$ (Chi-squared Test) $=57.91, \mathrm{df}($ degree of freedom) $=5, \mathrm{I}^{2}=91 \%, \mathrm{P}<0.1$, mean difference $(\mathrm{MD})=-9.21,95 \% \mathrm{CI}:-10.25$ to $-8.17, \mathrm{Z}=17.36, \mathrm{P}<0.05$. The non-surgical group showed a higher degree of pain and poorer ankle and hindfoot functions vs. the surgical group. Also, the pain degree of the non-surgical repair combined with surrounding tissue group was higher, and the ankle and hindfoot functions were poorer $v s$. the surgical repair treatment group. The Pain intensity results of the surgical and non-surgical groups were $\mathrm{Chi}^{2}=12.89, \mathrm{df}=2, \mathrm{I}^{2}=84 \%, \mathrm{P}<0.1, \mathrm{MD}=-9.51$, 95\% CI: -10.47 to $-8.55, \mathrm{Z}=19.39, \mathrm{P}<0.05$; the AOFAS scores of surgical repair treatment and non-surgical repair treatment were $\mathrm{Chi}^{2}=27.07, \mathrm{df}=6, \mathrm{I}^{2}=78 \%, \mathrm{P}<0.1, \mathrm{MD}=8.89,95 \% \mathrm{CI}: 8.26$ to 9.71, $\mathrm{Z}=24.30, \mathrm{P}<0.05$. It was revealed that surgical repair treatment can significantly reduce the postoperative pain of patients with ankle joint fractures, while the non-surgical repair treatment group had a higher degree of postoperative pain. The result was ( $M D=2.32,95 \%$ CI: 2.16 to $2.48, \mathrm{I}^{2}=100 \%, \mathrm{Z}=28.33, \mathrm{P}<0.05$ ).
\end{abstract}

Discussion: Surgical repair treatment of ankle fracture demonstrated significant curative effects, with fewer adverse reactions, and the stability of the ankle joint was well restored.

Keywords: Ankle fracture; surgical repair; special meta-analysis; talar tilt angle

Submitted May 31, 2021. Accepted for publication Aug 05, 2021.

doi: 10.21037/apm-21-1618

View this article at: https://dx.doi.org/10.21037/apm-21-1618

\section{Introduction}

Fractures are the most common clinical orthopedic presentation, among which joint fractures comprise the largest proportion. Ankle fractures account for about $4 \%$ of joint fractures. According to statistics from the World Medical Organization, ankle fractures are most common joint fracture, and the ankle is the most vulnerable joint of the human body (1). The ankle joint consists of only 3 bones, namely, the fibula, tibia, and talus. It is saddle- 
shaped and 1 of the most important weight-bearing joints (2). Studies have shown that ankle fractures usually occur in males younger than 50, while common in females over 50 years old. People generally believe that ankle fractures are caused by strenuous exercise. Interestingly, a sprain of the foot applying abnormal stress to the ankle joint, or insufficient bone strength to support normal load stress is the main cause of ankle fractures (3).

Clinically, ankle fractures are usually accompanied by local tissue injury (usually the deltoid ligament). Therefore, in this study, we focused on fractures combined with surrounding tissue injury. Literature has shown that nearly half of patients with ankle fractures are accompanied by deltoid ligament injury (4). As the most important weightbearing joint of the human body, if not treated in time, an ankle fracture will cause irreversible damage to the human body, such as traumatic arthritis and fracture nonunion. Sole reliance on the body's self-healing mechanism or improper treatment may result in abnormal fracture healing, and subsequent impairment of mobility.

Both surgical treatment and non-surgical treatment have their own advantages and disadvantages, but there is still a great controversy about the choice of specific treatment for clinical treatment of ankle fracture. This study innovatively searched Chinese and English references on the comparison of surgical and non-surgical treatment options for ankle joint injury since the establishment of the database for Meta-analysis. The aim of this study is to demonstrate that surgical treatment and non-surgical treatment have better clinical effects, and to provide evidence-based evidence for orthopedic surgeons when preparing treatment plans for patients with ankle fractures.

We present the following article in accordance with the PRISMA reporting checklist (available at https://dx.doi. org/10.21037/apm-21-1618).

\section{Methods}

\section{Literature retrieval}

Relevant studies were selected by logical Boolean search method, with "Fracture", "Ankles", "Fractured Foot", "Fractures Merge", and "Surgical Treatment" as search terms. The databases PubMed, Medline, Embase, China Biomedical Literature Database (CBM), HowNet, Wanfang, VIP, and Google Scholar were searched from their inception to 15 April 2021. The quality of the literature was evaluated using the software Review Manager
5.3 (Rev Man 5.3, Copenhagen: The Nordic Cochrane Center, The Cochrane Collaboration, 2014). Various search terms were combined freely, and the confirmed studies were traced using the search engine. Updated research progress was obtained after contacting experts in the field.

\section{Inclusion and exclusion criteria}

To be included, literature must have met all of the following criteria: (I) publicly published literature in Chinese and English; (II) patients aged 18-70 years and diagnosed with ankle fracture combined with surrounding tissue injury; (III) RCTs; (IV) intervention measures: the experimental group received surgical treatment, while the control group did not; (V) outcome indicators: Mazur score, American Orthopedic Foot and Ankle Society (AOFAS) score, visual analog scale (VAS), talus tilt angle, effective rate, medial malleolus gap, fracture healing time, adverse reactions. The exclusion criteria were as follows: (I) published literature not in Chinese or English; (II) non-RCTs; (III) duplicate literature, reviews, and descriptive case reports; (IV) no valid data provided; (V) research participants or data overlapped with each other; (VI) literature with too few experimental samples.

The abstract and the full text were independently screened by 2 senior experts, and 3 pre-experiments were needed prior to screening. Any inconsistencies were resolved by discussion or arbitration by a third expert.

\section{Quality assessment}

In the meta-analysis, the Newcastle-Ottawa Scale (NOS) of the Cochrane Collaboration was used to evaluate the pathological control studies, and the star system (out of 9 stars) was used to evaluate the participants, case comparison results, and group comparison results. References with 7 stars and above were considered high quality, that is, low risk bias; references with 1 star or no stars were considered low quality, that is, high risk bias; references with 2-6 stars were considered medium quality, that is, medium risk bias.

Quality evaluation of the references was independently conducted by 2 experts, and 3 pre-experiments were needed prior to the evaluation. Any inconsistencies were resolved by discussion or arbitration by a third expert.

\section{Data extraction}

A unified Excel table was used by 2 experts to collate the data independently, and 3 pre-experiments were needed 
prior to collation. Any inconsistencies were resolved by discussion or arbitration by a third expert. The following data were collated: (I) first author and the year of publication; (II) number of participants; (III) participant grouping: the experimental group and the control group; (IV) gender, age, research type, male to female ratio, number, various scores, medial malleolus gap, effective rate, talus tilt angle, fracture healing time, and adverse reactions.

\section{Statistical method}

The RevMan 5.3 software was used for meta-analysis.

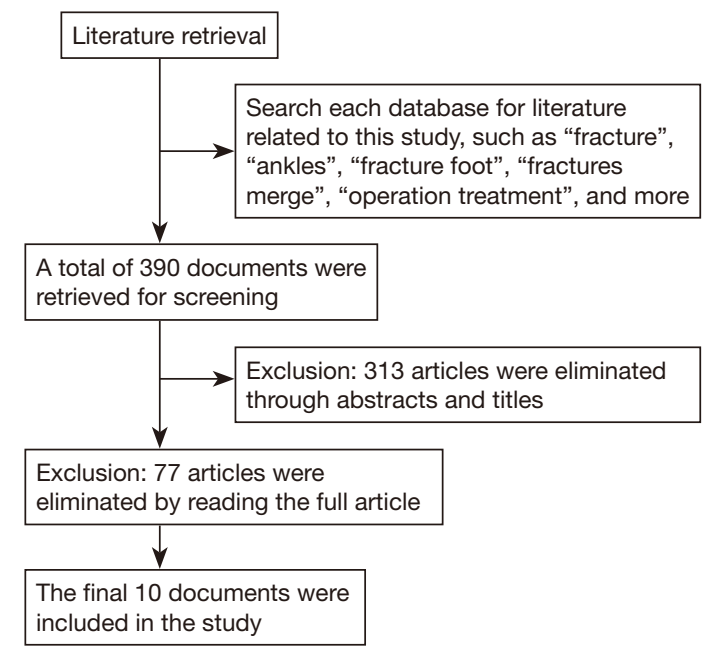

Figure 1 Flow chart depicting the screening process of the 10 RCTs. RCT, randomized controlled trial.
Mean difference (MD) or standardized mean difference (SMD), and 95\% confidence interval (CI) were used for the continuous variables for efficacy analysis. First, the included studies were tested for heterogeneity ( $\mathrm{Q}$ test). The risk of bias assessment adopted the table provided in the Rev Man software. Each effect was expressed using a 95\% CI. When $\mathrm{P}>0.1$ and $\mathrm{I}^{2}<50 \%$, the fixed effects model was used for meta-analysis. When $\mathrm{P}<0.1$ and $\mathrm{I}^{2}>50 \%$, the random effects model was used for meta-analysis.

\section{Results}

\section{Basic information of the included references and NOS rating results}

As shown in Figure 1, initially, a total of 390 references were included. After elimination of 313 articles through abstracts and titles, and further elimination of 77 articles through reading the full text of the article, 10 articles (5-13) were finally retained for meta-analysis. Literature was excluded mainly for these reasons: participants had only received conservative treatment and had not undergone surgical repair treatment; research information could not be extracted; other results indicators were used; there was a lack of original data. Table 1 shows the basic information of included literature. It can be noted that, all included literature was published between 1994 and 2020. The NOS rating results are shown in Figure 2. There were 4 references with 7 stars and above, 6 references with 2-6 stars, and 0 references with 1 star or 0 stars. Hence, they were all medium and high-quality references.

Table 1 Basic information of the included literature

\begin{tabular}{|c|c|c|c|c|c|}
\hline Author & Years & $\mathrm{N}(\mathrm{T} / \mathrm{C})$ & \multicolumn{2}{|c|}{ Age (years) } & Complete result data? \\
\hline Xia (5) & 2020 & $78(39 / 39)$ & $34.6 \pm 4.1$ & $36.2 \pm 4.3$ & Yes \\
\hline Novak (6) & 2019 & $104(52 / 52)$ & $36.2 \pm 8.3$ & $36.2 \pm 8.7$ & Yes \\
\hline Bali (7) & 2017 & $142(71 / 71)$ & $38.5 \pm 4.2$ & $38.3 \pm 4.3$ & Yes \\
\hline James (9) & 2013 & $48(24 / 24)$ & $45.6 \pm 9.2$ & $47.8 \pm 5.9$ & Yes \\
\hline Michelson (10) & 2012 & $160(80 / 80)$ & $44.4 \pm 6.4$ & $43.6 \pm 10.1$ & Yes \\
\hline Caschman (11) & 2004 & $96(48 / 48)$ & $50.2 \pm 5.9$ & $46.7 \pm 68$ & Yes \\
\hline Konrath (12) & 1997 & $24(12 / 12)$ & $37.9 \pm 3.2$ & $36.4 \pm 2.4$ & Yes \\
\hline
\end{tabular}




\section{Risk bias assessment results}

The multiple risk of bias assessment results by the Rev Man 5.3 software are shown in Figures 3 and 4. The bias risk assessment chart showed that the random sequence generation (selection bias), allocation hiding (selection bias), blind method of result evaluation (measurement bias), and incomplete result data (follow-up bias, selective reporting) were low-risk bias, and the bias risk of blind method (implementation bias) of participants and researchers was around $50 \%$.

\section{Mazur score}

The Mazur scores of the control group and the experimental group are shown in Figure 5. The Mazur score of the surgical group was significantly higher than that of the nonsurgical group, and the difference was significant. The result was $\mathrm{Chi}^{2}$ (Chi-squared Test) $=57.91 \mathrm{df}$ (degree of freedom) $=5, \mathrm{I}^{2}=91 \%, \mathrm{P}<0.1, \mathrm{MD}=-9.21,95 \% \mathrm{CI}:-10.25$ to -8.17 , $\mathrm{Z}=17.36, \mathrm{P}<0.05$. This indicated that patients with ankle joint fractures had a good gait after surgical repair treatment,

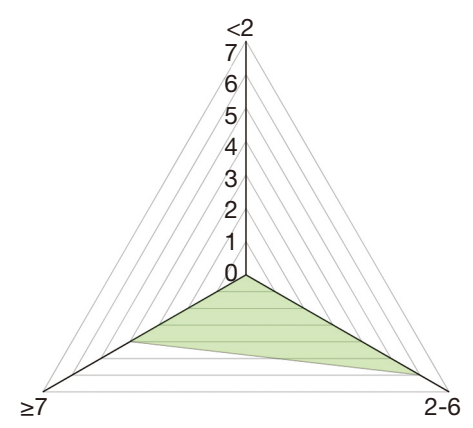

Figure 2 NOS rating results. NOS, Newcastle-Ottawa Scale. but the non-surgery group had a poor gait score.

A funnel chart showing the Mazur scores of the control group and the experimental group is presented in Figure 6. The circles included in the study were concentrated in the top area, and the accuracy was high. The circle is basically distributed on both sides of the midline and is roughly symmetric. Therefore, there was no publication bias in the included studies.

\section{AOFAS scores}

The AOFAS scores of the control group and the experimental group are shown in Figure 7. The score of the surgical group was significantly higher than that of the non-surgical group, and the score of the surgical repair combined with surrounding tissues was also significantly higher than the score of the non-surgical repair combined with surrounding tissue, with significant differences noted. The results of the surgical and non-surgical groups were $\mathrm{Chi}^{2}=12.89$, df $=2, \mathrm{I}^{2}=84 \%, \mathrm{P}<0.1, \mathrm{MD}=-9.51,95 \% \mathrm{CI}:-10.47$ to -8.55 , $\mathrm{Z}=19.39, \mathrm{P}<0.05$; the results of surgical repair treatment and non-surgical repair treatment were $\mathrm{Chi}^{2}=27.07, \mathrm{df}=6$, $\mathrm{I}^{2}=78 \%, \mathrm{P}<0.1, \mathrm{MD}=8.89,95 \%$ CI: 8.26 to $9.71, \mathrm{Z}=24.30$, $\mathrm{P}<0.05$. The pain level of the surgical group was lower than that of the non-surgical group, and the ankle and hindfoot functions were also significantly better than the non-surgical group (Figure 7). The non-surgical repair combined with surrounding tissue group exhibited a higher pain degree, and poorer ankle and hindfoot functions $v s$. the surgical repair combined with surrounding tissue group.

A funnel chart showing AOFAS scores between the control group and the experimental group is presented in Figure 8 . The circle is basically distributed on both sides of the midline and is roughly symmetric. Therefore, there was

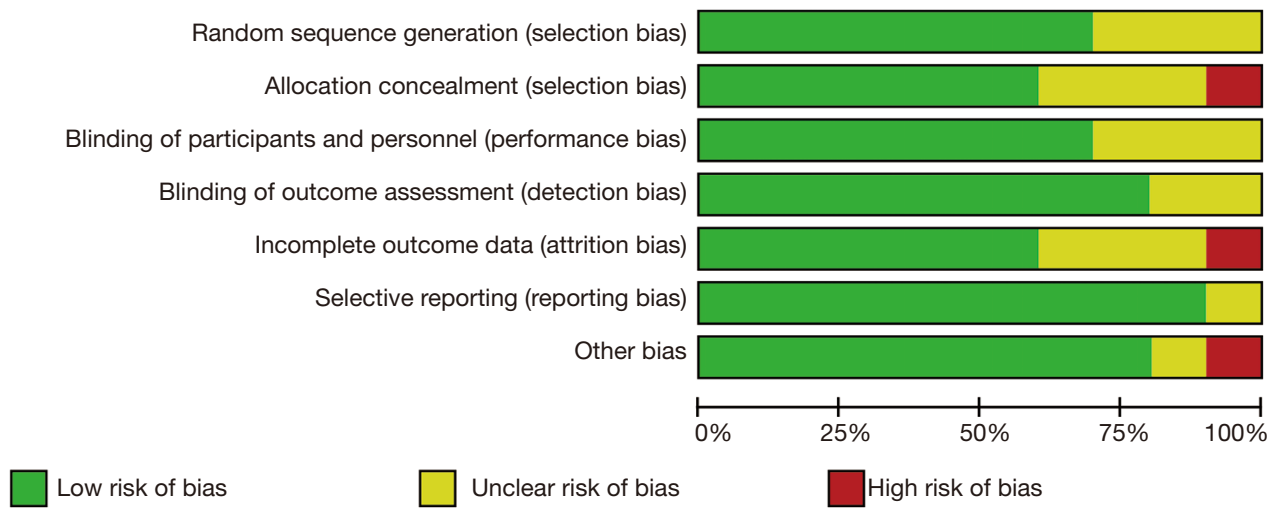

Figure 3 Risk of bias assessment results. 
no publication bias in the included studies.

\section{VAS score}

The VAS scores of the control group and the experimental group are shown in Figure 9. The surgical repair combined

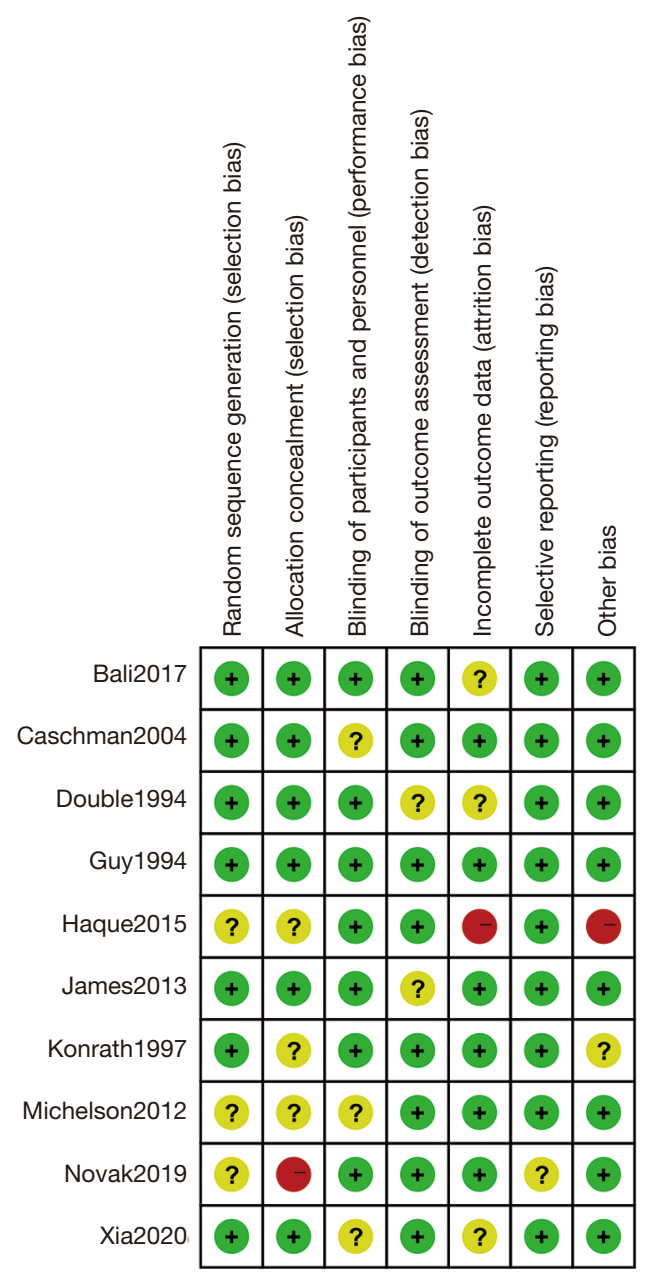

Figure 4 Multiple risk of bias assessment results. with surrounding tissue group had a higher score $v$ s. the non-surgical repair combined with surrounding tissue group, and the difference was significant. The result was df $=2, \mathrm{I}^{2}=100 \%, \mathrm{P}<0.1, \mathrm{MD}=2.32,95 \% \mathrm{CI}: 2.16$ to 2.48 , $\mathrm{Z}=28.33, \mathrm{P}<0.05$. Evidently, surgical repair can significantly reduce the postoperative pain of patients with ankle joint fractures combined with surrounding tissue injury.

A funnel chart showing the visual analog scores of the control group and the experimental group is displayed in Figure 10. The circles included in the study were concentrated in the top area, and the accuracy was high. The circle is basically distributed on both sides of the midline and is roughly symmetric. Therefore, there was no publication bias in the included studies.

\section{Effective rate}

The effective rates of the control group and the experimental group are shown in Figure 11. The effective rate of the surgical group was higher than that of the nonsurgical group, and the difference was significant. The result was $\mathrm{Chi}^{2}=2.88, \mathrm{df}=4, \mathrm{I}^{2}=0 \%, \mathrm{P}<0.1$, risk difference $(\mathrm{RD})=-0.16,95 \%$ CI: -0.22 to $-0.09, \mathrm{Z}=4.61, \mathrm{P}<0.05$. The effective rate of ankle fractures in the surgical group was significantly higher than that in the non-surgical group.

A funnel chart showing the effective rates of the control group and the experimental group is shown in Figure 12. The circles included in the study were concentrated in the top area, and the accuracy was high. The circle is basically distributed on both sides of the midline and is roughly symmetric. Therefore, there was no publication bias in the included studies.

\section{Talar tilt angle}

The talar tilt angles of the control group and the experimental group is shown in Figure 13. The surgical repair combined with surrounding tissue group showed a significantly larger talar tilt angle $v s$. the non-surgical repair combined with

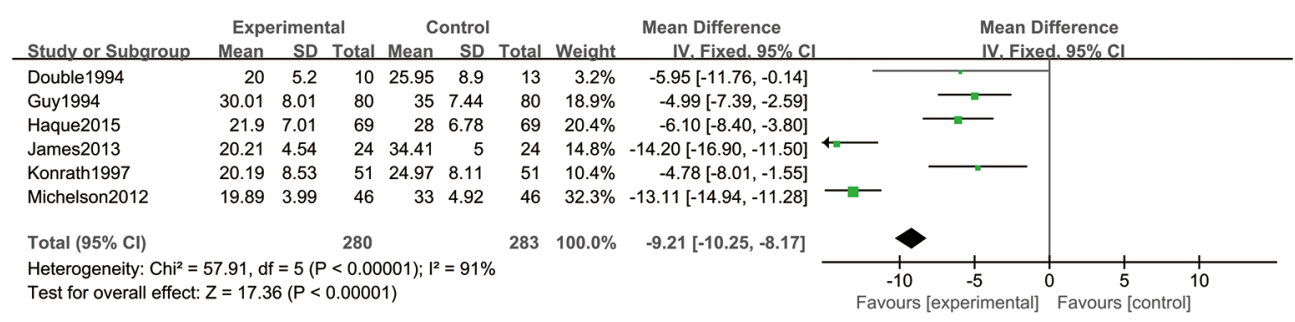

Figure 5 Forest plot showing the Mazur scores of the 2 participant groups. SD, standard deviation; CI, confidence interval. 


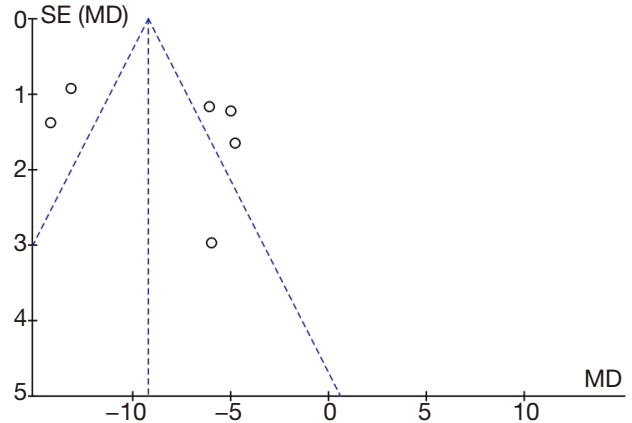

Figure 6 Funnel chart showing Mazur scores of 2 participant groups. surrounding tissue group, and the difference was significant. The result was $\mathrm{Chi}^{2}=139.36, \mathrm{df}=4, \mathrm{I}^{2}=97 \%, \mathrm{P}<0.1, \mathrm{MD}$ $=-5.45,95 \% \mathrm{CI}:-5.54$ to $-5.36, \mathrm{Z}=118.84, \mathrm{P}<0.05$. Hence, surgical repair can significantly improve the postoperative talus tilt angle of patients with ankle joint fractures combined with surrounding tissue injury.

A funnel chart showing the talar tilt angles of the control group and the experimental group is displayed in Figure 14. The circles of the included study were distributed on both sides of the midline and were roughly symmetrical, so there was no publication bias.

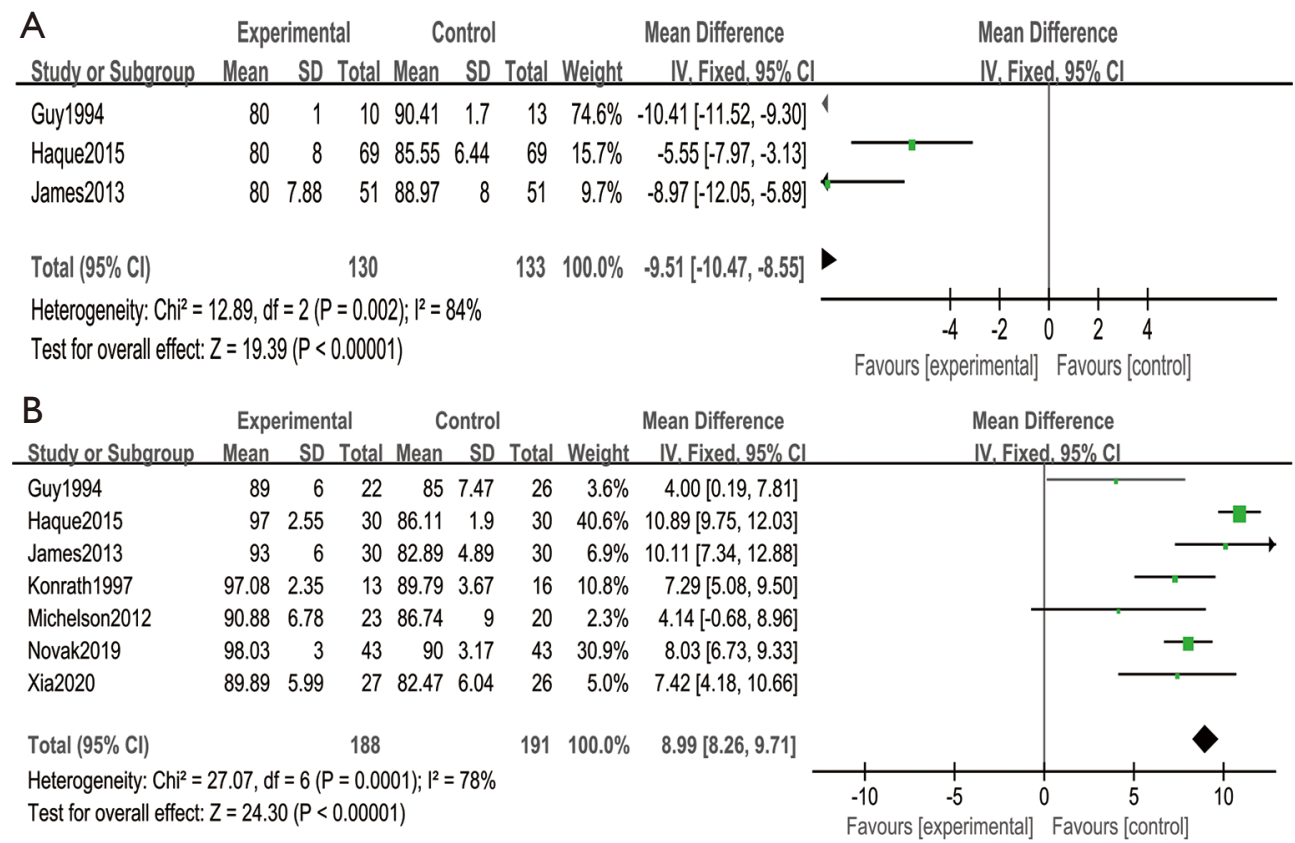

Figure 7 Forest plot showing the AOFAS scores of the 4 participant groups.
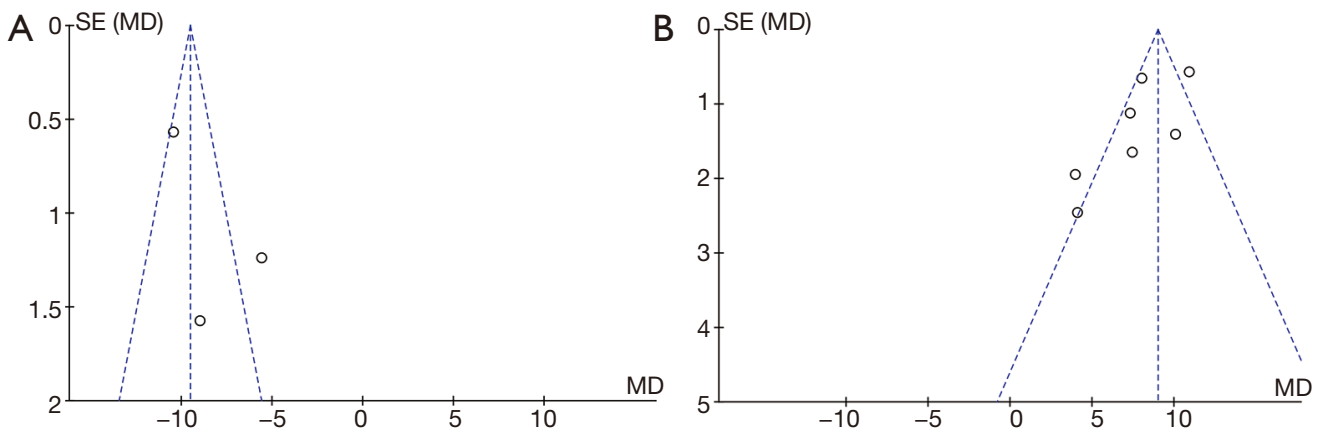

Figure 8 Funnel chart showing the AOFAS scores of the 4 participant groups. (A) Surgical group and non-surgical group; (B) surgical repair combined with surrounding tissue group and non-surgical repair combined with surrounding tissue group. AOFAS, American Orthopedic Foot and Ankle Society. 


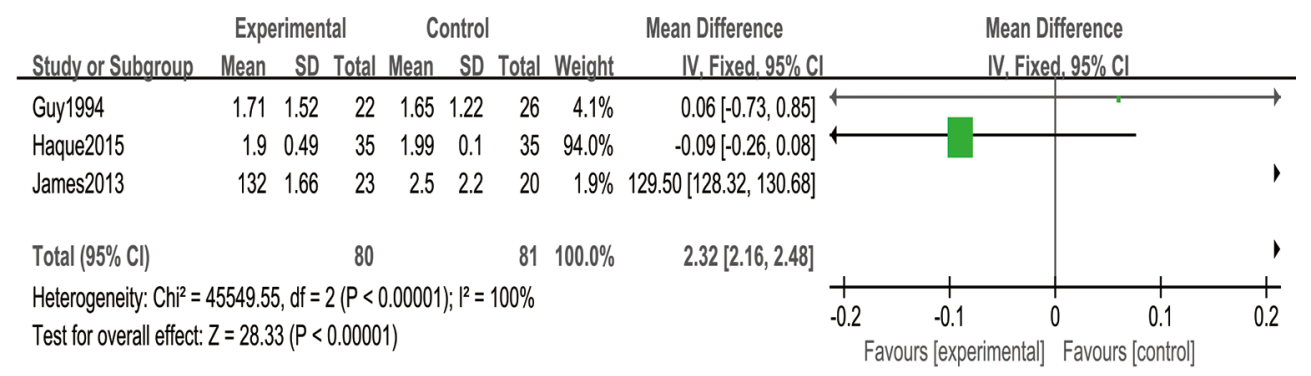

Figure 9 A forest plot showing the VAS scores of the 2 participant groups. VAS, visual analog scale; SD, standard deviation; CI, confidence interval.

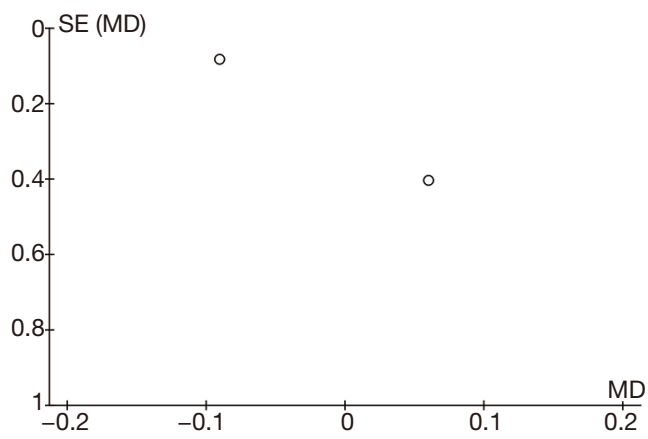

Figure 10 A funnel chart showing the VAS scores of the 2 participant groups. VAS, visual analog scale.

\section{Healing time}

The healing time of the control group and the experimental group is shown in Figure 15. The postoperative healing time in the surgical group was significantly shorter $v s$. the non-surgical group, and the difference was significant. The result was $\mathrm{Chi}^{2}=0.61, \mathrm{df}=3, \mathrm{I}^{2}=0 \%, \mathrm{P}<0.1, \mathrm{MD}=0.09$, $95 \%$ CI: -0.17 to $0.35, Z=0.65, P>0.05$. The surgical group showed shorter healing time compared to the non-surgical group.

A funnel chart showing the healing time of the control group and the experimental group is displayed in Figure 16. The circles of the included study were distributed on both sides of the midline and were roughly symmetrical, so there was no publication bias in the included study.

\section{Medial malleolus gap}

The medial malleolus gaps of the control group and the experimental group are shown in Figure 17. The medial malleolus gap in surgical repair combined with surrounding tissue group was significantly smaller than the non-surgical repair combined with surrounding tissue group, and the difference was significant. The result was $\mathrm{Chi}^{2}=0.00, \mathrm{df}=1$, $\mathrm{I}^{2}=0 \%, \mathrm{P}<0.1, \mathrm{MD}=-0.80,95 \% \mathrm{CI}:-1.14$ to $-0.46, \mathrm{Z}=4.57$, $\mathrm{P}<0.05$. Evidently, surgical repair can significantly reduce the medial malleolus gap of patients with ankle fractures combined with surrounding tissue injury.

A funnel chart showing the medial malleolus gaps between the control group and the experimental group is shown in Figure 18. The circles of the included study were distributed on both sides of the midline and were roughly symmetrical, so there was no publication bias in the included study.

\section{Adverse reaction}

The adverse reactions of the control group and the experimental group are shown in Figure 19. There were fewer adverse reactions in the surgical group $v s$. the nonsurgical group, and the surgical repair combined with surrounding tissue group also showed fewer adverse reactions $v s$. the non-surgical repair combined with surrounding tissue group, with significant differences noted. The results of surgical and non-surgical group were $\mathrm{Chi}^{2}$ $=3.60, \mathrm{df}=3, \mathrm{I}^{2}=17 \%, \mathrm{P}<0.1$, odds ratio $(\mathrm{OR})=3.75,95 \%$ 


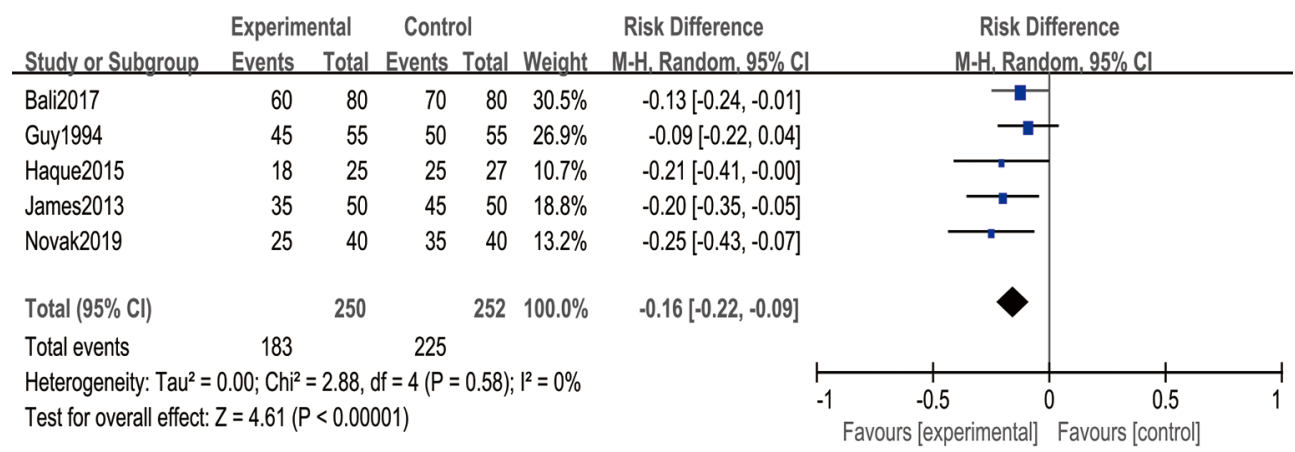

Figure 11 Forest plot showing the effective rates of 2 participant groups of ankle joint fractures. CI, confidence interval.

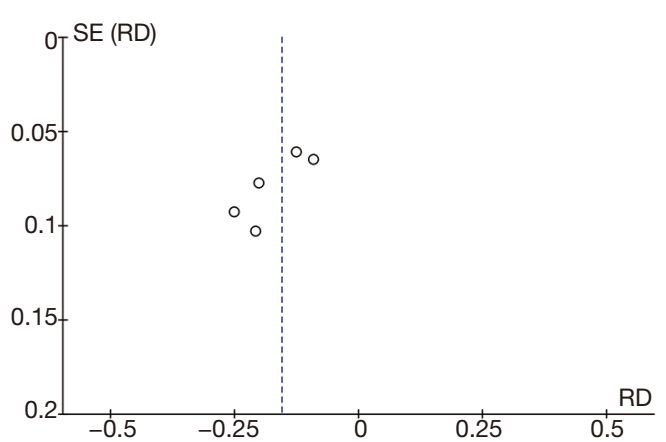

Figure 12 Funnel chart showing the effective rates of ankle fractures in the 2 participant groups.

CI: 1.61 to $8.73, \mathrm{Z}=3.07, \mathrm{P}<0.05$; the results of surgical repair treatment and non-surgical repair treatment group were $\mathrm{Chi}^{2}=0.58$, df $=3, \mathrm{I}^{2}=0 \%, \mathrm{P}<0.1, \mathrm{OR}=0.36,95 \%$ CI: 0.16 to $0.79, \mathrm{Z}=2.54, \mathrm{P}<0.05$. Obviously, the adverse reactions in the surgical group were fewer than those in the non-surgical group, and the adverse reactions in the nonsurgical repair group were fewer than those in the surgical repair group.
A funnel chart showing adverse reactions of the control group and the experimental group is displayed in Figure 20. The circle is basically distributed on both sides of the midline and is roughly symmetric. Therefore, there was no publication bias in the included studies.

\section{Discussion}

When it comes to orthopedic ankle fractures, there are many research reports, all of which clearly point out that surgical repair can effectively improve various indicators of patients with ankle fractures $(14,15)$. Studies have highlighted that as 1 of the 3 most important weightbearing joints of the human body, the ankle joint, once fractured or even combined with surrounding tissue injury, will seriously affect the overall stability of the body. At the same time, the reduction of the contact surface of the tibia leads to accumulated local stress in the foot, which further begets more serious consequences. At this time, patients with ankle fractures are very likely to develop degenerative joint disease (16). However, patients with ankle fractures

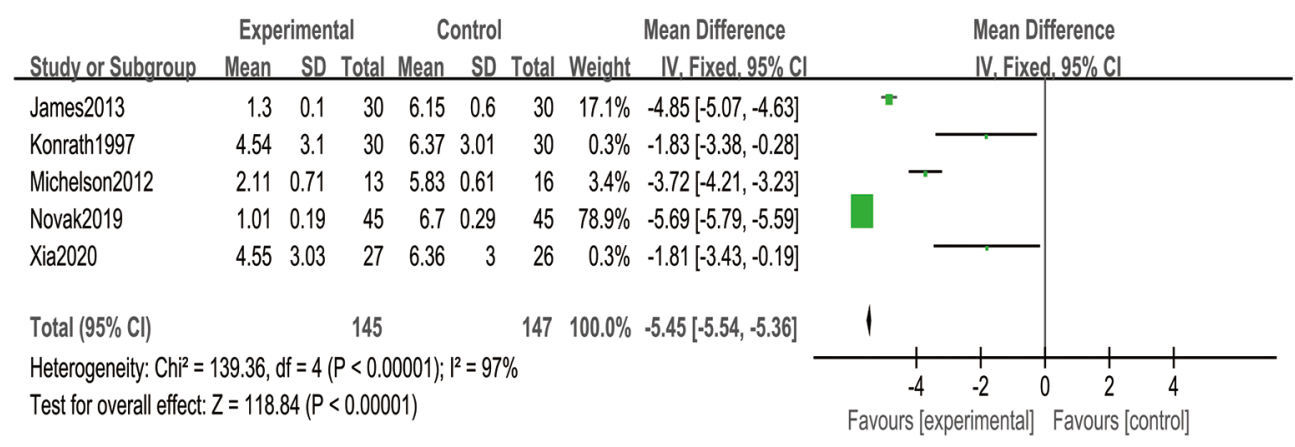

Figure 13 Forest plot showing the talar tilt angles of the 2 participant groups. SD, standard deviation; CI, confidence interval. 


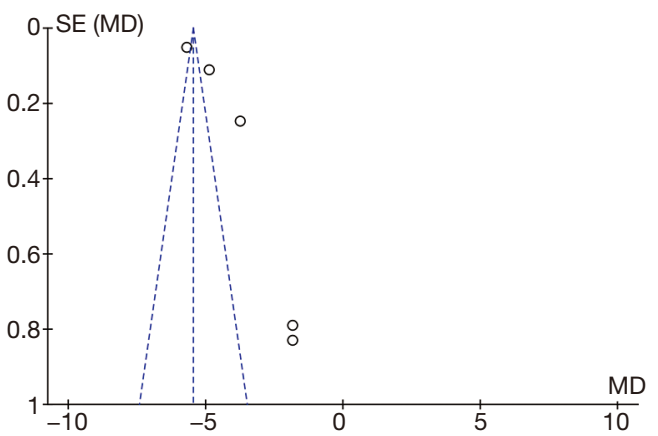

Figure 14 Forest plot showing the talar tilt angles of the 2 participant groups.

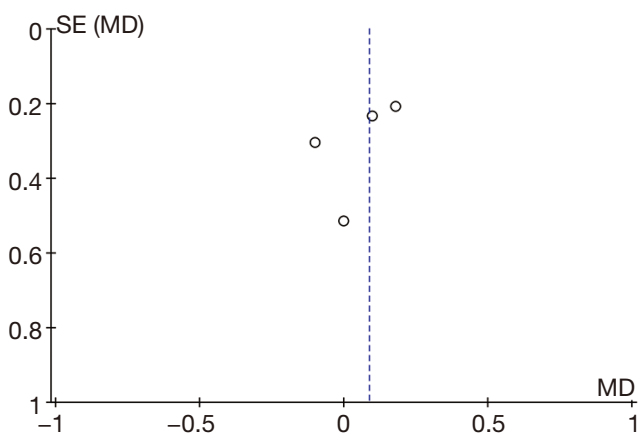

Figure 16 Funnel chart showing the healing time of the 2 participant groups.

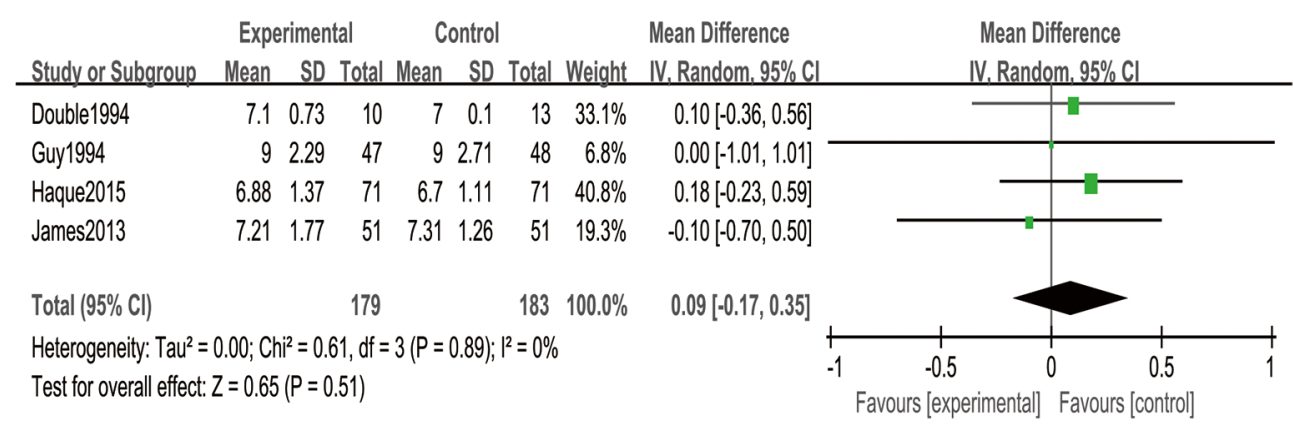

Figure 15 Forest plot showing the healing time of the 2 participant groups. SD, standard deviation; CI, confidence interval.

are mostly accompanied by advanced age, diabetes, and other conditions that are surgically contraindicated. Some studies have suggested that such patients are exempt from surgical repair treatment (17). However, some academics believe that if patients with ankle joint fractures promptly receive surgical repair, the ankle joint can be strengthened and fixed, which is conducive to fracture healing, and the rehabilitation exercise can be carried out as early as possible, which can effectively reduce adverse reactions such as muscle atrophy caused by long-term bed rest (18). The results of this research concur with this perspective.

According to some researchers, if an ankle fracture patient is accompanied by surrounding tissue injury, he can recover without surgical repair. Therefore, clinically, there is no consensus on whether surgical repair treatment is required. Some researchers hold that patients with ankle fractures combined with local tissue injury require surgical repair treatment for several reasons. One such reason is that patients who have not undergone surgical repair treatment experience persistent medial malleolus pain after surgery, and some even display ankle joint instability or even impaired ankle joint function. A second reason is that some patients develop traumatic arthropathy, and it is understood that the occurrence of traumatic arthropathy is due to unrepaired surrounding tissue injury (19-22).

\section{Conclusions}

We included 10 papers in this meta-analysis, and used the compound logic retrieval Boolean logic retrieval method to explore the efficacy of surgical repair treatment for patients with ankle fracture combined with surrounding tissue injury. The results revealed that surgical repair treatment can significantly reduce pain, shorten healing time, reduce the talar tilt angle of the medial malleolus, restore ankle joint stability, and reduce the occurrence of adverse reactions. 


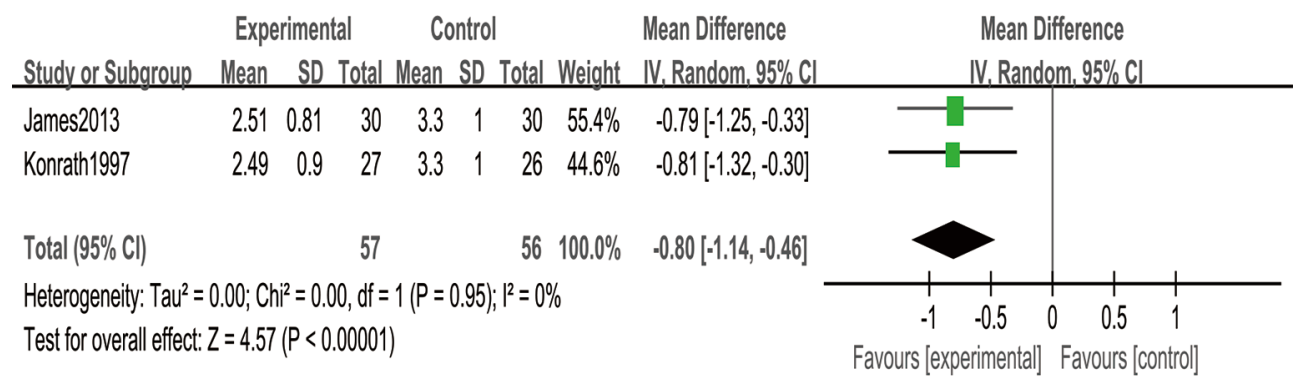

Figure 17 Forest plot showing the medial malleolus gaps between the 2 participant groups.

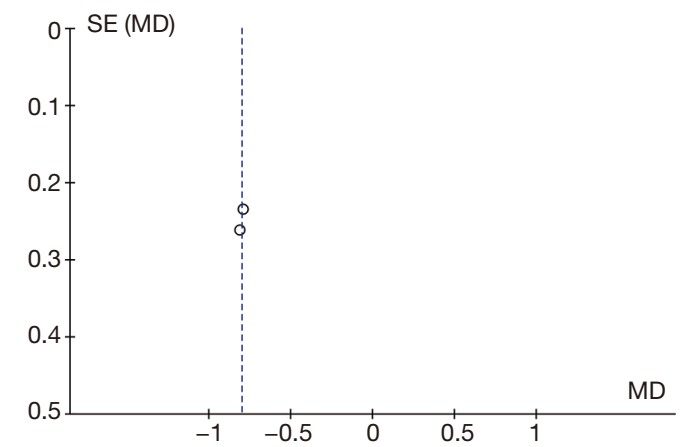

Figure 18 Funnel chart showing the medial malleolus gaps between the 2 participant groups.
However, there were some limitations to this study. The case-control included had bias itself, and the included literature was searched for manually, which would have omitted some literature. As a result, many risk factors and indicators may not have been included in the study, greatly reducing the combined effect size. The cases included were all international, and there was no local Chinese data included, which may have led to low reference value. In summary, this meta-analysis provides a theoretical basis and data support for surgical repair treatment of orthopedic ankle fractures combined with surrounding tissue injury, but its clinical efficacy requires further testing.

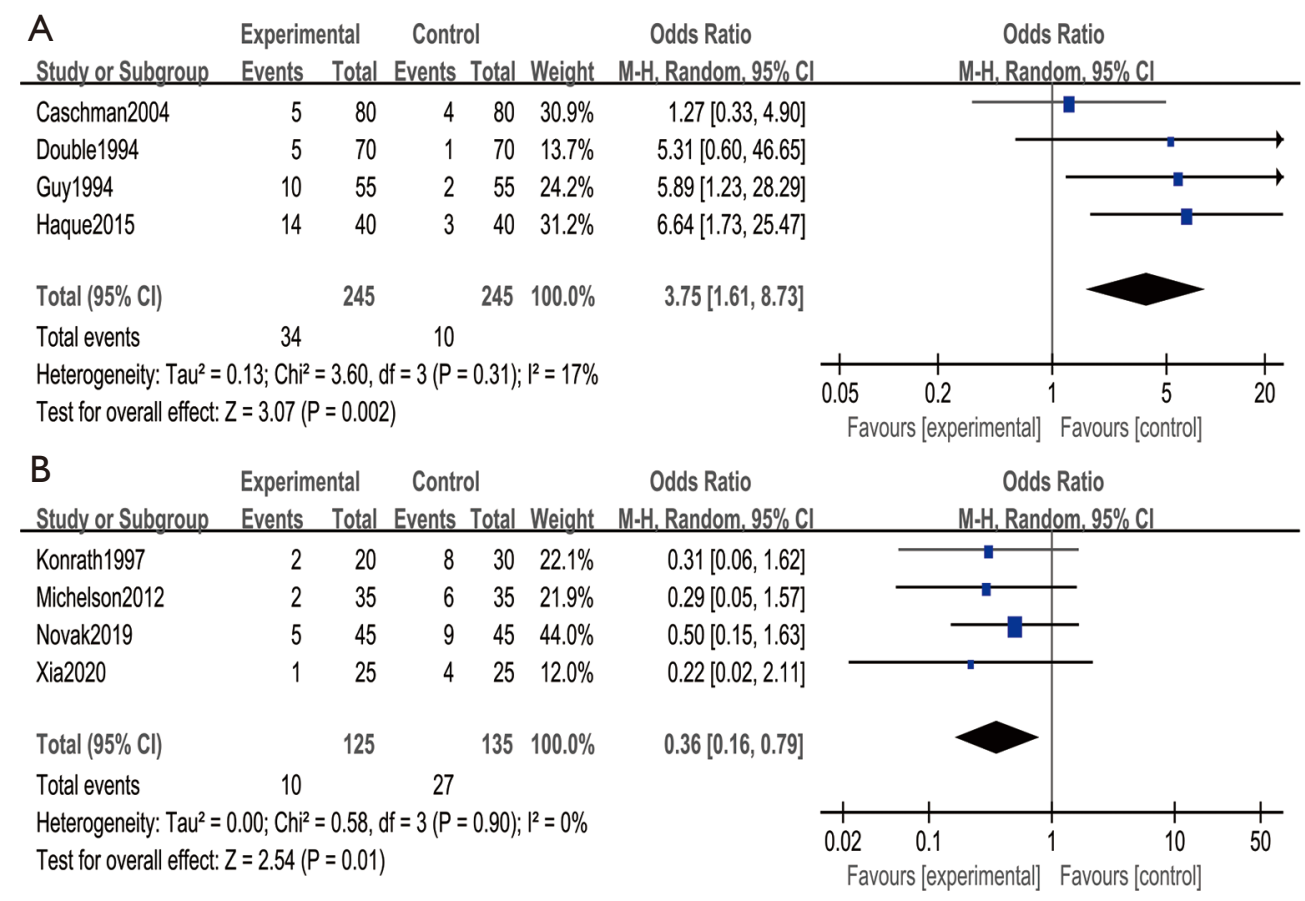

Figure 19 A forest plot showing the adverse reactions of the 4 participants. (A) Surgical group and non-surgical group; (B) surgical repair combined with surrounding tissue group and non-surgical repair combined with surrounding tissue group. CI, confidence interval. 

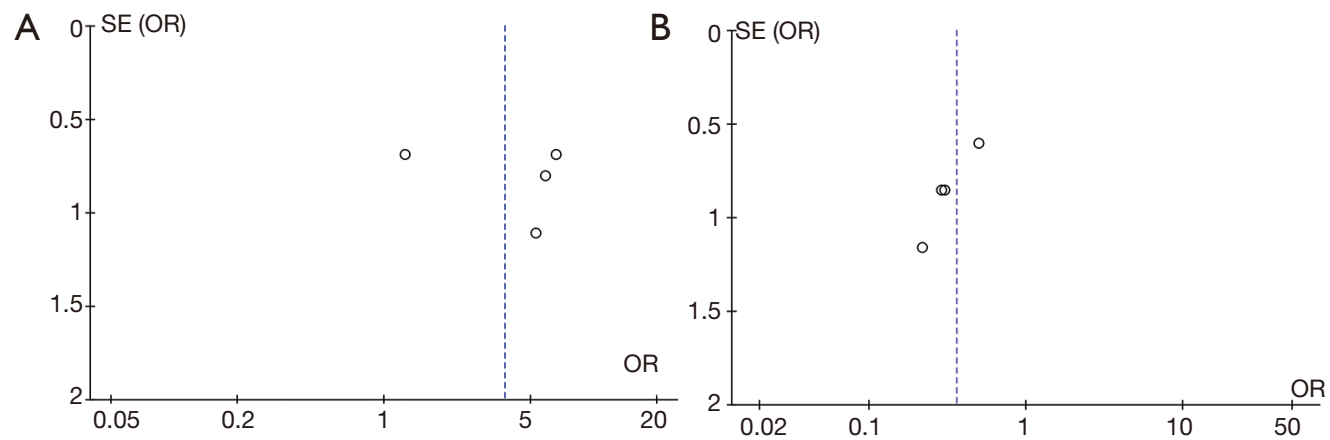

Figure 20 Funnel chart showing the adverse reactions of the 4 participant groups. (A) Surgical group and non-surgical group; (B) surgical repair combined with surrounding tissue group and non-surgical repair combined with surrounding tissue group.

\section{Acknowledgments}

Funding: None.

\section{Footnote}

Reporting Checklist: The authors have completed the PRISMA reporting checklist. Available at https://dx.doi. org/10.21037/apm-21-1618

Conflicts of Interest: All authors have completed the ICMJE uniform disclosure form (available at https://dx.doi. org/10.21037/apm-21-1618). The authors have no conflicts of interest to declare.

Ethical Statement: The authors are accountable for all aspects of the work in ensuring that questions related to the accuracy or integrity of any part of the work are appropriately investigated and resolved.

Open Access Statement: This is an Open Access article distributed in accordance with the Creative Commons Attribution-NonCommercial-NoDerivs 4.0 International License (CC BY-NC-ND 4.0), which permits the noncommercial replication and distribution of the article with the strict proviso that no changes or edits are made and the original work is properly cited (including links to both the formal publication through the relevant DOI and the license). See: https://creativecommons.org/licenses/by-nc-nd/4.0/.

\section{References}

1. Pakarinen HJ, Flinkkil TE, Ohtonen PP, et al. Stability criteria for nonoperative ankle fracture management. Foot
Ankle Int 2011;32:141-7.

2. Kusnezov NA, Eisenstein ED, Diab N, et al. Medial Malleolar Fractures and Associated Deltoid Ligament Disruptions: Current Management Controversies. Orthopedics 2017;40:e216-22.

3. Jensen SL, Andresen BK, Mencke S, et al. Epidemiology of ankle fractures. A prospective population-based study of 212 cases in Aalborg, Denmark. Acta Orthop Scand 1998;69:48-50.

4. Halai M, Jamal B, Rea P, et al. Acute fractures of the pediatric foot and ankle. World J Pediatr 2015;11:14-20.

5. Xia X, Yang Z, Deng C, et al. Effects of microsurgical repair treatment on the clinical efficacy, complications, and flap follow-up scores of patients with exposed steel plates after surgery for foot and ankle fractures. Ann Palliat Med 2020;9:4089-96.

6. Novak S. It's How You Present the Results: Commentary on an article by Kyle John Hancock, MD, et al.: "Efficacy of Multimodal Analgesic Injections in Operatively Treated Ankle Fractures. A Randomized Controlled Trial". J Bone Joint Surg Am 2019;101:e136.

7. Bali N, Aktselis I, Ramasamy A, et al. An evolution in the management of fractures of the ankle: safety and efficacy of posteromedial approach for Haraguchi type 2 posterior malleolar fractures. Bone Joint J 2017;99-B:1496-501.

8. Haque S, Davies MB. Oral thromboprophylaxis in patients with ankle fractures immobilized in a below the knee cast. Foot Ankle Surg 2015;21:266-8.

9. Michelson JD. Using decision analysis to assess comparative clinical efficacy of surgical treatment of unstable ankle fractures. J Orthop Trauma 2013;27:642-8.

10. Michelson J. Invited commentary: efficacy of popliteal block in postoperative pain control after ankle fracture 
fixation: a prospective randomized study. J Orthop Trauma 2012;26:562; discussion 562.

11. Caschman J, Blagg S, Bishay M. The efficacy of the A-V Impulse system in the treatment of posttraumatic swelling following ankle fracture: a prospective randomized controlled study. J Orthop Trauma 2004;18:596-601.

12. Konrath G, Moed BR, Watson JT, et al. Intramedullary nailing of unstable diaphyseal fractures of the tibia with distal intraarticular involvement. J Orthop Trauma 1997;11:200-5.

13. Paiement GD, Renaud E, Dagenais G, et al. Double-blind randomized prospective study of the efficacy of antibiotic prophylaxis for open reduction and internal fixation of closed ankle fractures. J Orthop Trauma 1994;8:64-6.

14. MacCormick LM, Baynard T, Williams BR, et al. Intraarticular Hematoma Block Compared to Procedural Sedation for Closed Reduction of Ankle Fractures. Foot Ankle Int 2018;39:1162-8.

15. Park CH, Kee HS, Kim BS, et al. Safety, compliance, and efficacy of newly developed videogame based exercises using wearable sensors for aged people. Ann Phys Rehabil Med 2018;61:e86-7.

16. Xie B, Yan S, Zhang H, et al. Clinical efficacy of locked fibular nail for the treatment of lateral malleolus fractures in elderly patients with diabetes. Zhongguo Gu Shang 2018;31:237-40.

Cite this article as: Xia X, Yang Z, Zhang Y, Deng C, Zhang W, Chen L. Systematic review and meta-analysis: surgical reparative treatment for orthopedic patients with ankle fracture complicated by peripheral tissue injury. Ann Palliat Med 2021;10(8):8869-8880. doi: 10.21037/apm-21-1618
17. Hernandez M, Rivkin G, Leibner ED, et al. Prevention of immobilization related muscular atrophy using the myospare device: a controlled, randomized, open study to investigate the feasibility, safety and efficacy of electrical gastrocneumius stimulation in ankle fractures. Orthopaedic Proceedings 2018;88-B(SII).

18. Saragas NP, Ferrao PN, Jacobson BF, et al. The benefit of pharmacological venous thromboprophylaxis in foot and ankle surgery. S Afr Med J 2017;107:327-30.

19. Wu K, Lin J, Huang J, et al. Evaluation of Transsyndesmotic Fixation and Primary Deltoid Ligament Repair in Ankle Fractures With Suspected Combined Deltoid Ligament Injury. J Foot Ankle Surg 2018;57:694-700.

20. Yde J, Kristensen KD. Ankle fractures: supinationeversion fractures of stage IV. Primary and late results of operative and non-operative treatment. Acta Orthop Scand 1980;51:981-90.

21. Ramsey PL, Hamilton W. Changes in tibiotalar area of contact caused by lateral talar shift. J Bone Joint Surg Am 1976;58:356-7.

22. Federici A, Sanguineti F, Santolini F. The closed treatment of severe malleolar fractures. Acta Orthop Belg 1993;59:189-96.

(English Language Editor: J. Jones) 